\title{
Patient Safety in Interventional Radiology: A CIRSE IR Checklist
}

\author{
M. J. Lee $\cdot$ F. Fanelli $\cdot$ P. Haage $\cdot$ K. Hausegger $\cdot$ \\ K. P. Van Lienden
}

Received: 27 July 2011/Accepted: 17 August 2011/Published online: 20 October 2011

(C) The Author(s) 2011. This article is published with open access at Springerlink.com

\begin{abstract}
Interventional radiology (IR) is an invasive speciality with the potential for complications as with other invasive specialities. The World Health Organization (WHO) produced a surgical safety checklist to decrease the morbidity and mortality associated with surgery. The Cardiovascular and Interventional Society of Europe (CIRSE) set up a task force to produce a checklist for IR. Use of the checklist will, we hope, reduce the incidence of complications after IR procedures. It has been modified from the WHO surgical safety checklist and the RAD PASS from Holland.
\end{abstract}

Keywords Clinical practice Patient safety .

Interventional radiology

Interventional radiology (IR) is an invasive speciality with the potential for complications, as with other invasive

\author{
M. J. Lee ( $\square)$ \\ Department of Academic Radiology, \\ Beaumont Hospital, Dublin 9, Ireland \\ e-mail: mlee@rcsi.ie \\ F. Fanelli \\ Vascular and Interventional Radiology Unit, \\ "Sapienza," University of Rome, Rome, Italy \\ P. Haage \\ Diagnostic and Interventional Radiology, \\ HELIOS Klinikum Wuppertal, Heusnerstr., Germany \\ K. Hausegger \\ Diagnostic and Interventional Radiology, \\ Landeskrankenanstalten-Betriebsgesellschaft-KABEG, \\ Klagenfurt, Germany \\ K. P. Van Lienden \\ Department of Interventional Radiology, \\ Academic Medical Center, Amsterdam, The Netherlands
}

specialities. Recently, the World Health Organization (WHO) produced a surgical safety checklist to decrease the morbidity and mortality associated with surgery. The Cardiovascular and Interventional Society of Europe (CIRSE) set up a task force to produce a checklist for IR. The checklist will, we hope, reduce the incidence of complications after IR procedures. The checklist has been modified from the WHO surgical safety checklist and the RAD PASS from Holland.

Safety has been an integral part of the civil aviation industry since its inception. Many factors have been recognised as contributing to airplane disasters. These include weather, engine failure, metal fatigue, bird strike, stalling, and fire, as well as, perhaps most importantly, human factors such as pilot error. The aviation industry has worked tirelessly to reduce pilot errors and in particular improper communications, which are often found to be factors in aircraft collision. The history of the development of the aviation safety checklist is of interest. In 1935, the Boeing Corporation and Douglas competed to manufacture long-range bombers for the U.S. Army. During testing of the new Boeing model 299 long-range bomber, one of the pilots forgot to release a lever that locked the elevator rudder controls. This resulted in a crash that resulted in two deaths. Douglas won the tender, delivering over a thousand aircraft to the U.S. Army Air Corps. However, Boeing persevered, and the U.S. Army Air Corps took a small number of their aircraft for further testing. It was at this time that U.S. Army pilots realized that this plane was not difficult to fly, but there were too many toggles and instruments for the human mind to remember. A group of test pilots got together and came up with the aviation safety checklist, which allowed them to fly the plane successfully. This plane eventually developed into the B17, which became one of the most famous heavy bombers of World War II. 
Recently, there have been a number of moves to introduce safety checklists into the world of medicine. The realization that one in every 150 patients admitted to hospital died because of an adverse event was the main driver behind the introduction of checklists [1]. In 2009, Haynes et al. [2] published the results of a study, which implemented a 19-item surgical safety checklist to determine whether this checklist would reduce complications and deaths associated with surgery. They chose eight hospitals in disparate locations with different health care systems and different economic circumstances. They compared data from 3,733 consecutively enrolled patients, before the introduction of the surgical safety checklist, with data on 3,955 consecutively enrolled patients after the introduction of the WHO surgical safety checklist. A significant reduction in the rate of death and complications occurred after the introduction of the surgical safety checklist. The death rate fell from $1.5 \%$ before the introduction of the checklist to $0.8 \%$ afterward. The complication rate fell from 11 to $7 \%$.

There was some criticism of this WHO-sponsored surgical safety checklist because it failed to control confounding factors such as concurrent implementation of outcomes measurement and feedback. The "surgical Hawthorne effect" was also a limitation, in that outcomes often improve when operators know that they are being evaluated. However, a further study by de Vries et al. [3] allowed for many of the confounding factors and produced a similar result. De Vries et al. evaluated a comprehensive checklist, which involved 11 different checklists dealing with issues such as preoperative preparation, intraoperative and postoperative care, the availability of imaging, informatory printed materials, patient site verification, communication of postoperative orders between caregivers, and discharge instructions. This comprehensive 100-item checklist completed by surgeons, anesthesiologists, nurses, and ancillary staff resulted in a drop in complications from 15.4 to $10.6 \%$ and a drop in mortality from 1.5 to $0.8 \%$. The study by de Vries makes it difficult to ignore the life-saving virtues of a safety checklist.

The introduction of a safety checklist for IR seems a reasonable next step, given the significant effect that the surgical safety checklist has had on surgical safety. Clearly, the incidence of complications, morbidity, and mortality in IR is much less than that of surgery because of its minimally invasive nature. However, complications do occur; in one study by Lewis et al. [4], a complication rate requiring vascular surgical intervention was found in $0.2 \%$ of 24,033 cardiac and vascular radiological procedures performed over a 13 -year period. Interestingly, $61 \%$ of these procedures were cardiac rather than IR procedures. Twenty-two patients had complications resulting from cardiac catheterization, and 40 patients had complications requiring vascular surgical procedures resulting from peripheral vascular intervention. There was a $5 \%$ reduction in risk for each successive year of observation in this study. In addition, patient contact before IR procedures is often quite short, and sometimes it is difficult for the interventionalist to gather all the necessary clinical information in a timely manner. Similarly, specific issues such as renal function and determination of allergic history is essential because most patients receive iodinated contrast medium. Last, the patient is usually sent back to the referring ward after the intervention where physicians might not always have an in-depth knowledge regarding the intervention and possible late complications. These facts beg the question whether a safety checklist in IR would avoid many of these complications and potential problems, not just in vascular intervention but throughout the varied IR retinue.

The advantage of a safety checklist for IR is that it ensures that human error in terms of forgetting key steps in patient preparation, intraprocedural care, and postoperative care are not forgotten. The Royal College of Radiologists has adapted the WHO surgical safety checklist for radiological interventions for England and Wales, and a group in Holland has been working on a RAD PASS Safety Checklist for IR (K. P. Van Lienden, personal communication). However, CIRSE thought that a more generic European-wide safety checklist for IR should be generated. A small task force was set up to achieve this. The safety checklist was drawn up communally with reference to the modified WHO IR safety checklist and the RAD PASS checklist. It is divided into three sections (Appendix).

The first section is titled "Procedure Planning." It is envisaged that this should be completed by the IR nurse/ ward nurse. The preprocedure checklist contains important items such as whether or not the patient is receiving anticoagulation medication, whether the patient is allergic to contrast material, and whether the patient has abnormal renal function requiring prophylaxis for contrast-induced nephropathy. These are items that can be easily forgotten on a busy day in the interventional suite, but their omission could result in potentially disastrous complications for the patient. It is hoped that adoption of the checklist will ensure that all of these items are recognized ahead of time and dealt with appropriately.

The second section of the checklist is a sign-in section, which can be completed by the IR resident, nurse, or staff interventional radiologist, and which deals with immediate checks that should be performed when the patient is in the IR room. This includes items such as checking that the patient is the correct patient, and that the correct side and site are being operated on.

The third section is entitled "Sign-out" and should be completed by the interventional radiologist who performed the procedure. The sign-out section encompasses patient orders, follow-up tests, and appointments made.

The checklist was trialed in four European hospitals and modified to achieve the final version. The checklist was not 
subject to rigorous scientific analysis because we thought that the surgical safety checklist it is based on has already proven to be efficacious. Moreover, we live in times where patients, caregivers, and third-party payers demand high-quality care with minimal risk and complication rates. CIRSE hopes that the widespread adoption of this CIRSE IR checklist will enhance patient safety throughout Europe and beyond, and strongly recommends this checklist to all practicing interventional radiologists. The checklist was designed to be general so it can be applied to a wide variety of interventional procedures. We realize that this IR safety checklist may not suit every interventional radiologist and every center or IR procedure, but it can be modified to suit any IR practice. It is available to download from the CIRSE Web site and is being translated into different European languages. On behalf of CIRSE, we hope it enhances patient safety in IR practice.

Conflict of interest The authors declare that they have no conflict of interest.

Open Access This article is distributed under the terms of the Creative Commons Attribution Noncommercial License which permits any noncommercial use, distribution, and reproduction in any medium, provided the original author(s) and source are credited.

\section{References}

1. de Vries EN, Ramrattan MA, Smorenburg SM et al (2008) The incidence and nature of in-hospital adverse events: a systematic review. Qual Saf Health Care 17:216-233

2. Haynes AB, Weiser TG, Berry WR et al (2009) A surgical safety checklist to reduce the morbidity and mortality in a global population. N Engl J Med 360:491-499

3. de Vries EN, Hollmann MW, Smorenburg SM et al (2009) Development and validation of the Surgical Patient Safety System (SURPASS) checklist. Qual Saf Health Care 18:121-126

4. Lewis DR, Bullbulia RA, Murphy P et al (1999) Vascular surgical intervention for complications of cardiovascular radiology: 13 years experience in a single centre. Ann R Coll Surg Engl $81: 23-26$

\section{Appendix}

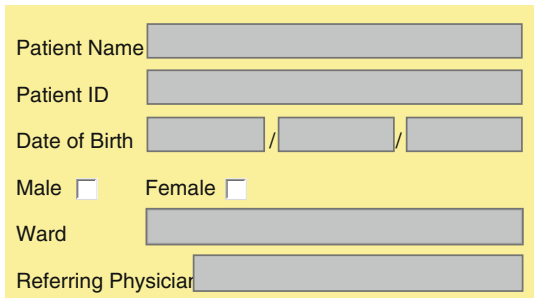

\section{PROCEDURE PLANNING}

Discussed referring Physician/MDT

Imaging Studies Reviewed

Relevant Medical History

Informed Consent

CIN Prophylaxis

Specifc Tools Present/Ordered

Fasting Order Given

Relevant Lab Tests Ordered

Anaesthesiologist Necessary

Anticoagulant Medication Stopped

Postinterventional (ICU) Bed Required

Contrast Allergy Prophylaxis Necessar

Name

Signature

\begin{tabular}{|c|c|c|}
\hline YES & NO & $\mathrm{N} / \mathrm{A}$ \\
\hline & $\Gamma$ & $\Gamma$ \\
\hline & $\Gamma$ & $\Gamma$ \\
\hline & $\Gamma$ & $\Gamma$ \\
\hline & $\Gamma$ & \\
\hline & Г & $\Gamma$ \\
\hline & & $\Gamma$ \\
\hline & & Г \\
\hline & & \\
\hline & Г & $\Gamma$ \\
\hline & $\Gamma$ & $\Gamma$ \\
\hline & 厂 & $\Gamma$ \\
\hline & & \\
\hline
\end{tabular}

\section{CIRSE IR Patient Safety Checklist*}

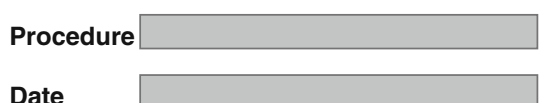

Date

Cardiovascular and Interventional Radiological Society of Europe

\section{RSE}

\begin{tabular}{|c|c|c|c|}
\hline SIGN IN & YES $N$ & NO & N/A \\
\hline All team members introduced & $\Gamma$ & Г & \\
\hline All Records with Patient & $\Gamma$ & $\Gamma$ & Г \\
\hline Correct patient/side/site & $\Gamma$ & Г & \\
\hline Patient Fasting & $\Gamma$ & Г & Г \\
\hline IV Access & Г & Г & Г \\
\hline Monitoring Equipment Attached & $\Gamma$ & Г & Г \\
\hline Coagulation screen/Lab Tests checkec & & $\Gamma$ & Г \\
\hline Allergies and/or Phrophylaxis Checked & $d$ & Г & \\
\hline Antibiotics/other drugs administered & $\Gamma$ & $\Gamma$ & Г \\
\hline Consent/Complications Discussed & Г & $\Gamma$ & \\
\hline
\end{tabular}

Signature

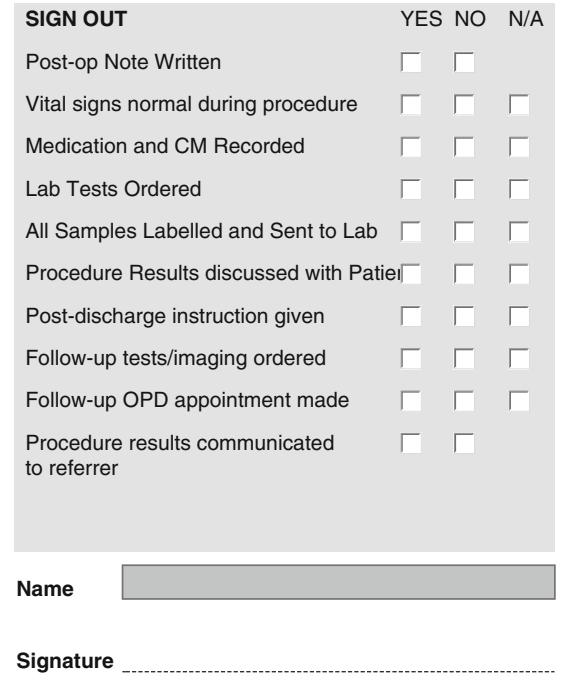

* Modified from RADPASS \& WHO SURGICAL CHECKLIST 